\title{
The Role of Surface Infiltration in Hydromechanical Coupling Effects in an Unsaturated Porous Medium of Semi-Infinite Extent
}

\author{
L. Z. Wu, ${ }^{1}$ A. P. S. Selvadurai, ${ }^{2}$ and J. Yang $^{1}$ \\ ${ }^{1}$ State Key Laboratory of Geohazard Prevention and Geoenvironment Protection, Chengdu University of Technology, \\ Chengdu, Sichuan 610059, China \\ ${ }^{2}$ Department of Civil Engineering and Applied Mechanics, McGill University, Montreal, QC, Canada H3A 2 K6
}

Correspondence should be addressed to L. Z.Wu; cewulz@gmail.com

Received 20 June 2017; Revised 20 September 2017; Accepted 15 October 2017; Published 23 November 2017

Academic Editor: Marco Petitta

Copyright (c) 2017 L. Z. Wu et al. This is an open access article distributed under the Creative Commons Attribution License, which permits unrestricted use, distribution, and reproduction in any medium, provided the original work is properly cited.

\begin{abstract}
Rainfall infiltration into an unsaturated region of the earth's surface is a pervasive natural phenomenon. During the rainfall-induced seepage process, the soil skeleton can deform and the permeability can change with the water content in the unsaturated porous medium. A coupled water infiltration and deformation formulation is used to examine a problem related to the mechanics of a two-dimensional region of semi-infinite extent. The van Genuchten model is used to represent the soil-water characteristic curve. The model, incorporating coupled infiltration and deformation, was developed to resolve the coupled problem in a semi-infinite domain based on numerical methods. The numerical solution is verified by the analytical solution when the coupled effects in an unsaturated medium of semi-infinite extent are considered. The computational results show that a numerical procedure can be employed to examine the semi-infinite unsaturated seepage incorporating coupled water infiltration and deformation. The analysis indicates that the coupling effect is significantly influenced by the boundary conditions of the problem and varies with the duration of water infiltration.
\end{abstract}

\section{Introduction}

Coupling between water infiltration and mechanical deformation in partially saturated porous media is central to many natural and man-made systems in civil and environmental engineering. During water infiltration the porewater pressure is redistributed, on one hand by the hydraulic properties of the partially saturated soil including retention characteristics and permeability and on the other hand by the external loading due to climate conditions (rainfall intensity, duration, and evapotranspiration rate). Changes in the porewater pressure are generated by infiltration, which in turn modifies the hydraulic domain and induces deformations of the partially saturated porous medium. Alternatively, any variation in the mechanical loading can exert an effect on the infiltration process. It is indeed the hydromechanical coupled response of a partially saturated porous medium that is responsible for the most common instabilities associated with water infiltration: landslides and excessive settlements, due to collapse or shear strength reduction $[1,2]$.

The prediction of the hydromechanical behavior of geomaterials due to rainfall infiltration plays an important role in addressing geotechnical and geoenvironmental issues related to ground movement or landslides and other geologic disasters. Examples of the effects of saturation variation induced by wetting on the soil deformation have been reported at both laboratory and in situ scales $[1,3,4]$.

Analytical solutions play an important role in the examination of water infiltration in saturated and unsaturated heterogeneous porous media [5-13]. Srivastava and Yeh [5] derived an analytical approach to describe one-dimensional rainfall infiltration towards the water table. In recent years, analytical solutions to the one-dimensional problem incorporating rainfall-induced hydromechanical coupling in unsaturated porous media have been developed $[11,14,15]$. 
The interactions between infiltration and deformation in porous media have been modelled computationally using various multiphysics schemes. Numerical techniques for analyzing coupled water infiltration and deformation in partially saturated porous media are being increasingly utilized because rainfall infiltration involves complicated geometries, soil heterogeneity, and complex spatial and temporal boundary conditions [16]. Analytical approaches to such practical situations are difficult [17]. Many numerical approaches that incorporate the coupled seepage and deformation behaviors involved in water infiltration into a partially saturated porous medium have been presented in the literature in both the soil mechanics and geotechnical disciplines [18]. Numerical models have also been developed to investigate the coupled hydromechanical behavior of partially saturated soils where ground deformation occurs as a result of the water table fluctuation and surface loading [19-21] or the development of soil slope instability [22, 23]. Multiphase, coupled elastoviscoplastic finite element analysis has been used to examine the evolution of pore-water pressure and deformations due to rainwater infiltration into a one-dimensional soil column [17].

A semi-infinite unsaturated extension to the $2 \mathrm{D}$ coupled problem is seldom considered when the effects of both seepage and deformation in a partially saturated porous media are being investigated. The numerical method presented in this paper examines rainfall infiltration into a twodimensional semi-infinite region that also incorporates the coupled seepage and deformation in the unsaturated porous medium. The two-dimensional computational model was developed to resolve the semi-infinite infiltration coupled with deformation in partially saturated porous media. The numerical solution is verified by an analytical solution for the 1D coupled infiltration, and the effect of semi-infinite boundaries on the coupling is analyzed. We then use these numerical approaches to examine the hydromechanical coupling in a 2D semi-infinite unsaturated porous medium.

\section{Governing Equations for Coupled Seepage and Deformation in Unsaturated Soils}

In the development of a coupled unsaturated seepage and deformation model we use the following assumptions: (i) the soil fabric is deformable, the grains are nondeformable, and the water in the pore space is incompressible; (ii) hysteresis of the soil-water characteristic curve is neglected; (iii) the pore-air pressure within the porous medium is assumed to be constant.

2.1. Static Equilibrium Equations. The equation of equilibrium for a soil mass can be written as

$$
\sigma_{i j, j}+f_{i}=0
$$

where $\sigma_{i j}$ is the total stress tensor and $f_{i}$ is the body force vector.

A thermodynamically consistent effective stress equation has been developed in terms of the effective stress tensor $\sigma_{i j}^{\prime}$ in the unsaturated porous medium $[24,25]$. On the basis of Bishop's effective stress, the effective stress for a partially saturated porous medium, related to the skeletal deformation, can be given as [26]

$$
\sigma_{i j}^{\prime}=\sigma_{i j}-\left[u_{w} S_{r}+u_{a}\left(1-S_{r}\right)\right] \delta_{i j}
$$

where $u_{a}$ is the pore-air pressure; $u_{w}$ is the pore-water pressure; $S_{r}$ is the degree of saturation; $\delta_{i j}$ is Kronecker's delta function.

The soil skeleton is assumed to exhibit linear isotropic elastic behavior; a constitutive equation can be written as [27]

$$
\varepsilon_{i j}=\frac{\sigma_{i j}^{\prime}}{2 G}-\frac{\lambda \delta_{i j} \sigma_{k k}^{\prime}}{2 G(3 \lambda+2 G)},
$$

where $\varepsilon_{i j}$ is the strain tensor; $G=\mu=E / 2(1+\nu) ; \lambda=E v /(1+$ $\nu)(1-2 \nu)$ where $G$ is the shear modulus, $\nu$ is Poisson's ratio of the soil, and $E$ is the elastic modulus; $\mu$ and $\lambda$ are Lamé's constants.

The linearized strain-displacement relations are

$$
\varepsilon_{i j}=\frac{1}{2}\left(\frac{\partial u_{i}}{\partial x_{j}}+\frac{\partial u_{j}}{\partial x_{i}}\right)
$$

in which $u_{i}, u_{j}$ are displacement components.

We restrict our attention to the class of problems where the deformation corresponds to a state of plane strain. From assumption (iii), the pore-air pressure is a constant and therefore the partial derivative of the pore-air pressure with respect to time is zero. The external stress applied to the soil mass is assumed to remain constant with time (no loading or the applied loading is unchanged). Making use of (2) to (3), the partial derivative of the volumetric strain can be obtained as

$$
\frac{\partial \varepsilon_{v}}{\partial t}=\frac{-2}{C} \frac{\partial\left(S_{r} u_{w}\right)}{\partial t}
$$

in which $\varepsilon_{v}$ is the volumetric strain and $C=E /(1+v)(1-2 v)$.

2.2. Soil-Water Characteristic Curve and Hydraulic Conductivity. If the van Genuchten model [28] is used to describe the soil-water characteristic curve (SWCC), we can use the following expression for the volumetric moisture content:

$$
\theta=\theta_{r}+\frac{\theta_{s}-\theta_{r}}{\left[1+(\alpha h)^{n}\right]^{m}},
$$

where $h$ is the pressure head, the pore-water pressure $u_{w}=$ $\gamma_{w} h, \gamma_{w}$ is the unit weight of water, $\alpha, n$, and $m$ are fitting parameters, $m=1-1 / n, \theta_{r}$ is the residual volume moisture content, and $\theta_{s}$ is the saturated moisture content.

The unsaturated hydraulic conductivity can be expressed as

$$
k=k_{s} S_{e}^{1 / 2}\left[1-\left(1-S_{e}^{1 / m}\right)^{m}\right]^{2}
$$

in which $k_{s}$ is the coefficient of infiltration at saturation and $S_{e}=1 /\left[1+(a h)^{n}\right]^{m}$ is the effective saturation. 
2.3. Seepage Equations. The two-dimensional Richards' equation is employed, which, when combined with Darcy's Law for an unsaturated medium, gives

$$
\frac{\partial}{\partial x}\left(k \frac{\partial h}{\partial x}\right)+\frac{\partial}{\partial z}\left[k\left(\frac{\partial h}{\partial z}-1\right)\right]=\frac{\partial \theta}{\partial t}
$$

where $\theta$ is the volumetric water content; $k$ is the coefficients of hydraulic conductivity in the principal directions $x$ and $z$; $t$ is the time variable. (Implicit in (8) is the assumption of isotropy of the porous medium.) The hydraulic properties of the porous medium are assumed to be isotropic, and the elastic deformability characteristics of the porous medium are considered as isotropic.

The change in the volumetric water content in an unsaturated porous medium is related to the normal stress or strain and the suction of the partially saturated porous medium. Dakshanamurthy et al. [29] proposed that the constitutive equation for an unsaturated porous medium be expressed as

$$
\theta=\beta \varepsilon_{v}+\omega\left(u_{a}-u_{w}\right)
$$

in which $\left(u_{a}-u_{w}\right)$ is the matric suction and the coefficients $\beta$ and $\omega$ are used to express the volume change modulus $\beta=$ $E / H(1-2 \nu)$ and $\omega=1 / R-3 \beta / H$, where $H$ is the elastic modulus for the water phase with respect to the effective stress [24]; $R$ is the elastic modulus for the water phase with respect to matric suction $\left(u_{a}-u_{w}\right)$ [29]; $1 / R$ is the envelope of the soil-water characteristic curve.

Assuming that $H$ is a constant, we obtain from the van Genuchten model [28]

$$
\begin{aligned}
\frac{1}{R} & =\frac{\partial \theta}{\partial h} \frac{\partial h}{\partial \psi} \\
& =\left(-\frac{1}{\rho_{w} g}\right)\left(\theta_{s}-\theta_{r}\right) m \alpha n S_{e}^{1+1 / m}(-\alpha h)^{n-1}
\end{aligned}
$$

in which $\psi$ is the matric suction.

We now take the partial derivative of (10) with respect to time $t$ and substitute the result into (6), to obtain the following equation:

$$
\frac{\partial \theta}{\partial t}=-\rho_{w} g\left[\frac{2 \beta}{C}\left(S_{r}+h \frac{\partial S_{r}}{\partial h}\right)+\omega+h \frac{\partial(1 / R)}{\partial h}\right] \frac{\partial h}{\partial t} .
$$

Thus, the right hand side of (11) is a function of the pressure head.

Referring to a Cartesian coordinate system and substituting (11) into (9), we obtain the equation governing the transient hydromechanical process in a partially saturated porous medium as follows:

$$
\begin{aligned}
& \frac{\partial}{\partial x}\left(k_{x} \frac{\partial h}{\partial x}\right)+\frac{\partial}{\partial z}\left[k_{z}\left(\frac{\partial h}{\partial z}-1\right)\right] \\
& \quad=-\rho_{w} g\left[\frac{2 \beta_{0}}{C}\left(S_{r}+h \frac{\partial S_{r}}{\partial h}\right)+\omega_{0}+h \frac{\partial(1 / R)}{\partial h}\right] \frac{\partial h}{\partial t} .
\end{aligned}
$$

If the porous soil mass is considered to be rigid $(C \rightarrow \infty)$, (12) reduces to the unsaturated flow equation without coupling between infiltration and deformation in the unsaturated porous medium.

\section{Analytical Solution to the Coupled Infiltration in a Semi-Infinite Region}

The effective analysis of unsaturated seepage coupled with deformation in a semi-infinite unsaturated domain is based on the following assumptions: (a) the soil is homogeneous and behaves as an isotropic elastic material; (b) the soil structure is deformable, but the pore water is not compressible; (c) the volume change of the soil is due to the wetting or drying of the soil only; (d) the coefficient of permeability at full saturation is kept constant regardless of ground deformation; (e) the pore-air pressure in the soil remains constant.

Based on Darcy's Law and conservation of the 1D fluid mass, combined with the stress-strain relationship applicable to normal strains proposed by Fredlund and Rahardjo [24], the $1 \mathrm{D}$ governing equation for partially saturated infiltration coupled with deformation in a semi-infinite domain, where the water compressibility is neglected, can be given as $[11,14]$

$$
\frac{\partial}{\partial z}\left[k(h) \frac{\partial(h-z)}{\partial z}\right]=\left[n \frac{\partial S_{r}}{\partial h}+S_{r} \alpha_{c} \frac{(1+\nu) \gamma_{w}}{(1-v) H}\right] \frac{\partial h}{\partial t},
$$

where $\gamma_{w}$ is the unit weight of water; $n$ is porosity; $\alpha_{c}=1-$ $K / K_{s}$ is the hydromechanical coupling coefficient $\left(0 \leq \alpha_{c} \leq\right.$ 1 ), where $K$ is the bulk modulus of the solid skeleton and $K_{s}$ is the bulk modulus of the soil solids; and $v$ is Poisson's ratio.

The constitutive behavior of unsaturated soils that allow fluid infiltration is bound to have advanced concepts involving plasticity and dilatant plasticity in their descriptions. If such processes take place, then the alterations in the fluid transport characteristics should also be accounted for. It seems unlikely that an elastoplastic solution could be developed through analytical solutions to address these issues. The linearization of the modelling including elastic linearized model is a useful first step towards understanding the coupling effects. Ideally, developments involving both saturated and unsaturated geomaterials should take into account the irreversible deformations of the porous skeleton, which can include both elastoplasticity and creep [30, 31]. The latter processes are, however, less likely to be significant in the type of problems discussed in this paper. Elastoplasticity and poromechanical effects can be important and in order to examine such effects the problem must be formulated using a three-dimensional incremental approach with specified failure criteria and flow rules $[32,33]$. The elementary elasticity approach is not perfect but will provide an opportunity to assess whether further extensions to other constitutive modelling will be warranted. The approach will also provide a bound for the behavior of the soil under rainfall influx.

The van Genuchten model [28] is complex and highly nonlinear. Hence the model is difficult to apply to analytical methods. The exponential variation of hydraulic conductivity with pore-water pressure was applied by Gardner [34]. In many analytical solutions, the relatively simple exponential hydraulic conductivity function of Gardner [34] is employed [12]. The relationship between pore-water pressure and hydraulic conductivity is nonlinear. Combining this result with Kirchoff's transformation and observing that pore-water pressure varies linearly with hydraulic conductivity after a series of transformations allow the linearization of Richards' 
equation for transient partially saturated flow in order to achieve an analytical solution to coupled infiltration and deformation in unsaturated soils. Therefore, $k(h)=k_{s} e^{\alpha h}$ and $\theta(h)=\theta_{s} e^{\alpha h}$ are employed to describe the hydraulic conductivity of an unsaturated porous medium and the relationship between the volumetric moisture and matric suction. $\alpha$ is the desaturation coefficient.

Substituting the exponential forms into (8), we obtain the $1 \mathrm{D}$ governing equation:

$$
\frac{\partial}{\partial z}\left[k_{s} e^{\alpha h} \frac{\partial(h-z)}{\partial z}\right]=P \cdot e^{\alpha h} \frac{\partial h}{\partial t}
$$

in which $P=\theta_{s} \alpha+\gamma_{w} \alpha_{c}(1+\nu) /(1-\nu) H$.

If coupling is not considered, (14) becomes

$$
\frac{\partial}{\partial z}\left[k_{s} e^{\alpha h} \frac{\partial(h-z)}{\partial z}\right]=\alpha \theta_{s} \cdot e^{\alpha h} \frac{\partial h}{\partial t}
$$

The infiltration equations for a semi-infinite domain can be solved with a specified surface flux $q(t)$. The initial porewater pressure is described by $h(z, 0)=h_{r}$, and a boundary condition is $\left.h(z, t)\right|_{z \rightarrow \infty}=h_{r}$.

Dimensional variables of $Z=\alpha z, T=\alpha^{2} k_{s} t / P$, and $K(Z$, $T)=e^{\alpha h} \cdot e^{T / 4-T / 2}$ are employed. Based on (14) and (15), we obtain

$$
\begin{aligned}
\frac{\partial^{2} K}{\partial Z^{2}} & =\frac{\partial K}{\partial T} \\
K(Z, 0) & =e^{\alpha h_{r}} e^{-Z / 2}=f(Z) \\
{\left[-\frac{\partial K}{\partial Z}+\frac{K}{2}\right]_{z=0} } & =\frac{q}{k_{s}} e^{T / 4}=g(T) \\
\left.K(Z, T)\right|_{Z \rightarrow \infty} & =e^{\alpha h_{r}} e^{T / 4} .
\end{aligned}
$$

The analytical solution to (16) is given by [35]

$$
\begin{aligned}
& K(Z, T)=\int_{\beta=0}^{\infty} M(\beta, X) \\
& \cdot e^{-\beta^{2} t}\left[\bar{F}(\beta)+\int_{t^{\prime}=0}^{T} e^{\beta^{2} t^{\prime}} A\left(\beta, t^{\prime}\right) d t^{\prime}\right] d \beta
\end{aligned}
$$

in which

$$
\begin{aligned}
\bar{F}(\beta) & =\int_{z=0}^{\infty} M\left(\beta, z^{\prime}\right) f\left(z^{\prime}\right) d z^{\prime} \\
A\left(\beta, t^{\prime}\right) & =\left.M(\beta, z)\right|_{z=0} g\left(t^{\prime}\right) \\
M(\beta, Z) & =\sqrt{\frac{2}{\pi}} \frac{\beta \cos \beta Z+0.5 \sin \beta Z}{\sqrt{\beta^{2}+0.25}},
\end{aligned}
$$

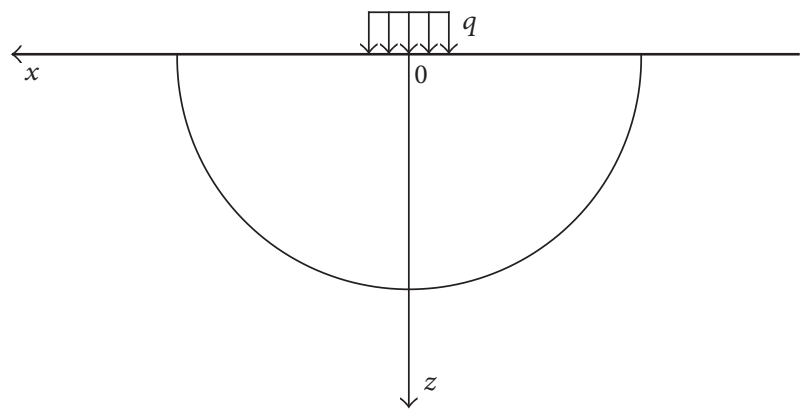

FIGURE 1: Water infiltration into a semi-infinite unsaturated porous medium.

where $f\left(z^{\prime}\right)$ and $g\left(t^{\prime}\right)$ represent the initial and surface boundary conditions, respectively; $M(\beta, Z)$ is the kernel of the equation.

\section{Computational Modelling of Water Infiltration in a Semi-Infinite Unsaturated Region}

Figure 1 shows a two-dimensional semi-infinite region where there is rainfall influx over a symmetric finite region. The $2 \mathrm{D}$ semi-infinite region is represented by a symmetric model of finite extent shown in Figure 2; in this model the region $A \rightarrow 0$ represents the width of the rainfall region with different rainfall intensities and the region $\mathrm{A} \rightarrow \mathrm{B}$ is an impervious boundary. The two sides $0 \mathrm{D}$ and $\mathrm{BC}$ are controlled by a zero water flow rate in the horizontal direction. The lower boundary CD is controlled by $\nabla h=0$, and the entire domain is imposed with zero normal displacement boundary conditions on the base of the region and along the vertical sides. An external stress $(200 \mathrm{kPa})$ is vertically applied at the surface boundary $0 \mathrm{~B}$ in Figure 2.

The domain shown in Figure 2 is discretized into 478 triangular finite elements using an adaptive mesh feature available in COMSOL Multiphysics ${ }^{\circledR}$. For the computational simulations, the model dimensions are set to $w$ in length by $d$ in height, and the width of the rainfall region is $a$, as shown in Figure 2. In Figure 2, $u$ denotes displacement, and $\tau$ is the shear stress. Since the COMSOL ${ }^{\mathrm{TM}}$ mesh generation is adaptive, stabilized finite element methods are necessary to develop numerical results to examine the two-dimensional symmetric coupled seepage and deformation problem related to a partially saturated porous medium [36]. In the multiphysics code COMSOL the mesh refinement is automatically adjusted when the wetting front caused by water infiltration moves into the partially saturated porous medium [36].

In the model, the two lateral boundaries shown in Figure 2 are subjected to fluid flow boundary condition corresponding to a zero flux and constant head boundary condition as follows:

$$
\begin{aligned}
{\left[k(h) \frac{\partial h}{\partial x}\right]_{x=0} } & =0 \quad 0<z<d \\
{[h]_{x=w} } & =h_{r} \quad 0<z<d .
\end{aligned}
$$




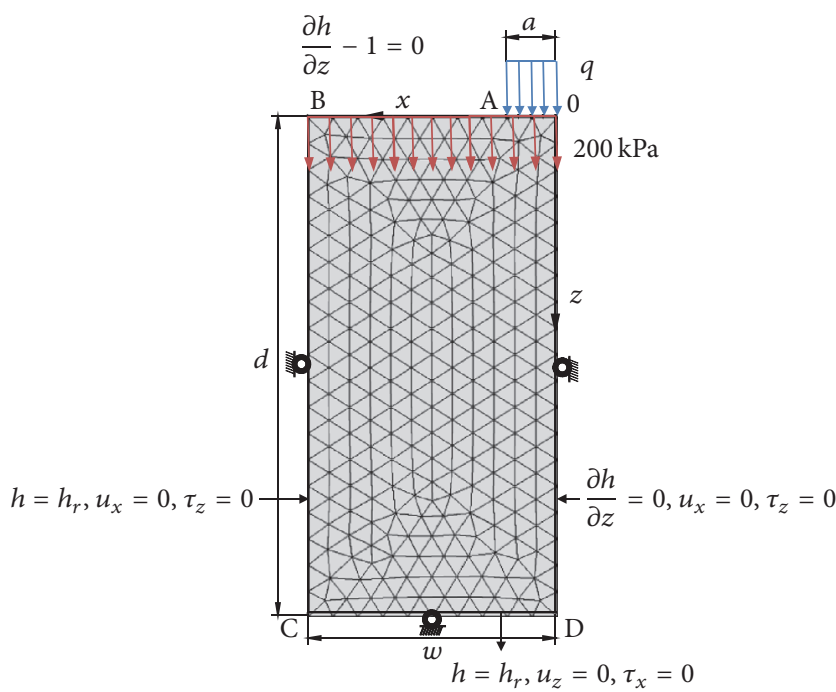

FIgURE 2: The symmetric two-dimensional model and the finite element mesh used in the computational modelling.

At the plane of symmetry the fluid flow boundary conditions consist of the lower and upper boundaries, which can be given as

$$
\begin{aligned}
h(x, 0,0) & =h_{r} \\
{\left[k(h) \frac{\partial h}{\partial z}-k(h)\right]_{z=d} } & =q(x, t) \quad 0<x<w,
\end{aligned}
$$

where $h_{r}$ is the pore-water pressure head that corresponds to the residual volumetric water content and $q$ is a function which varies with position $(x)$ and time $(t)$. In addition, the boundary conditions corresponding to traction and displacements applicable to the porous medium are

$$
\begin{aligned}
& \left.u(x, z, t)\right|_{x=0, z \in[0, d]}=0 \\
& \left.u(x, z, t)\right|_{x=w, z \in[0, d]}=0 \\
& \left.u(x, z, t)\right|_{z=d, x \in[0, w]}=0 .
\end{aligned}
$$

Initially the medium is assumed to be undeformed; the only free boundary is at the ground surface, and the other three boundaries are controlled by a fixed constraint. The initial pressure head distribution (i.e., the initial condition) is written as

$$
h(x, z, 0)=h_{i}(x, z),
$$

where the initial pressure head $h_{i}$ is assumed to be constant.

The model of a semi-infinite domain is depicted in Figure 1. The top flux is specified when $z$ approaches infinity, and the pore-water pressure head is constant $\left(h_{r}\right)$. The initial porewater pressure is $h_{r}$. The parameters used are listed in Table 1. Figure 3 compares the 1D numerical and analytical solutions for the finite unsaturated domain considering the coupling between infiltration and deformation. The $1 \mathrm{D}$ numerical solution is obtained by substituting $k(h)=k_{s} e^{\alpha h}$ and

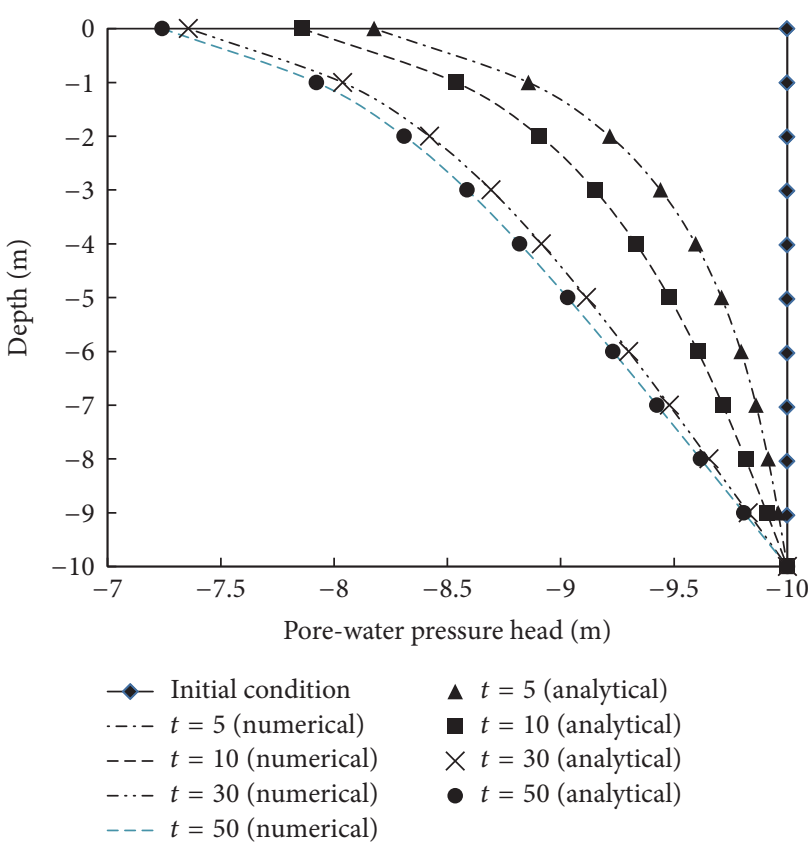

FIGURE 3: A comparison between the numerical and analytical solution to the $1 \mathrm{D}$ coupled infiltration problem.

$\theta(h)=\theta_{s} e^{\alpha h}$ into (8), which, when combined with (4), can be resolved in COMSOL. The difference observed between the analytical and numerical solutions to the $1 \mathrm{D}$ infiltration with coupled effects is small.

The parameters for the partially saturated porous medium used in this study are listed in Tables 2 and 3. Table 2 gives the parameters used in the calculations involving the partially saturated porous medium. The basic soil properties are presented in Table 3.

Figure 4 shows the variation of pressure head with duration of rainfall under the conditions of coupled water infiltration and deformation in an unsaturated porous medium. In Figure $4, w=10 \mathrm{~m}, d=10 \mathrm{~m}$, and $a=1 \mathrm{~m}$. The changes in the pressure head occur only in the shallow layers of the partially saturated porous medium. Noticeable changes in the pore-water pressure head occur close to the water infiltration region.

As shown in Figures 5 and 6, the pressure head and displacement at the upper boundary $0 \mathrm{~B}$ change with the duration of rainfall. The parameters are the same as in Figure 4. At the start of water infiltration, the pressure head and the ground settlement is uniform. As rainfall continues, water infiltrates into the deeper regions of the unsaturated porous medium and uneven displacement in the shallow layers of the unsaturated soil begins to occur. After $t=10 \mathrm{~h}$, we observe different displacements at the ground surface of the unsaturated region, as shown in Figure 6. With continued rainfall, differential settlements occur at the surface of the partially saturated porous medium.

Figure 7 shows how changing the infiltration width $0 \mathrm{~B}$ affects the pore-water pressure head at the boundary $\mathrm{OB}$ shown in Figure 2. In Figure 7 the infiltration width is altered $(w=5,10,15,20,25,30 \mathrm{~m})$ but the other dimensions 
TABLE 1: The parameters used in analytical solutions to $1 \mathrm{D}$ unsaturated infiltration.

\begin{tabular}{|c|c|c|c|c|}
\hline $\begin{array}{l}\text { Saturated volumetric water } \\
\text { content }\left(\theta_{s}\right)\end{array}$ & $\begin{array}{l}\text { Coefficient of permeability at } \\
\text { saturation }\left(k_{s}\right)\end{array}$ & Rainfall intensity $(q)$ & $\begin{array}{l}\text { Desaturation } \\
\text { coefficient }(\alpha)\end{array}$ & $\begin{array}{l}\text { Elastic modulus with } \\
\text { respect to suction } \\
\text { change }\end{array}$ \\
\hline 0.4 & $1 \times 10^{-5} \mathrm{~m} / \mathrm{s}$ & $10^{-5} \mathrm{~m} / \mathrm{s}$ & $0.01 \mathrm{~cm}^{-1}$ & $10 \mathrm{MPa}$ \\
\hline
\end{tabular}

TABLE 2: The parameters used in the calculations involving SWCC.

\begin{tabular}{lccccc}
\hline $\begin{array}{l}\text { Saturated volumetric water } \\
\text { content }\left(\theta_{s}\right)\end{array}$ & $\begin{array}{c}\text { Residual volumetric water } \\
\text { content }\left(\theta_{r}\right)\end{array}$ & Initial porosity $\left(n_{0}\right)$ & $a$ & $n$ & $\begin{array}{c}\text { Rainfall intensity } \\
(q)\end{array}$ \\
\hline 0.4 & 0.01 & 0.35 & 0.02 & 2 & 0.5 \\
\hline
\end{tabular}

TABLE 3: The basic soil properties of an unsaturated porous medium.

\begin{tabular}{lccc}
\hline Elastic modulus & Poisson's ratio & Unit weight of soil mass & Unit weight of water \\
\hline $10 \mathrm{MPa}$ & 0.3 & $18.0 \mathrm{kN} / \mathrm{m}^{3}$ & $10.0 \mathrm{kN} / \mathrm{m}^{3}$ \\
\hline
\end{tabular}

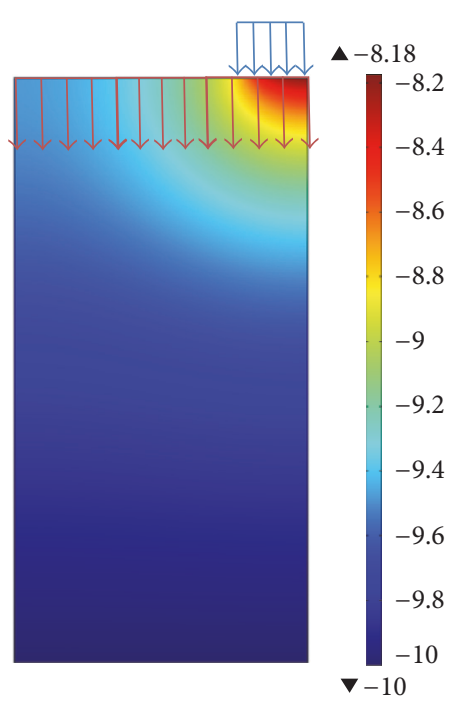

(a)

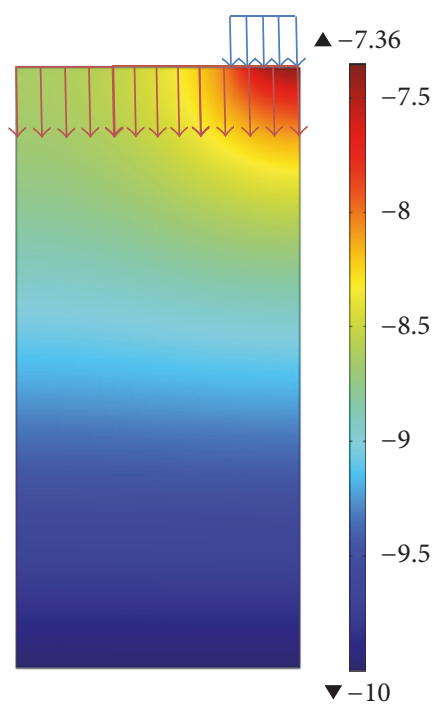

(c)

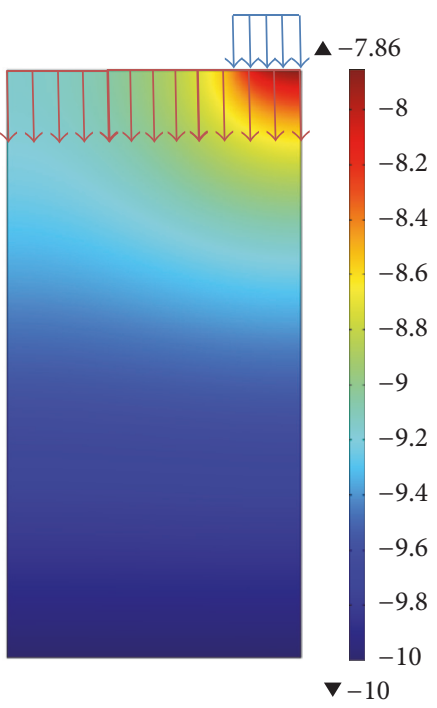

(b)

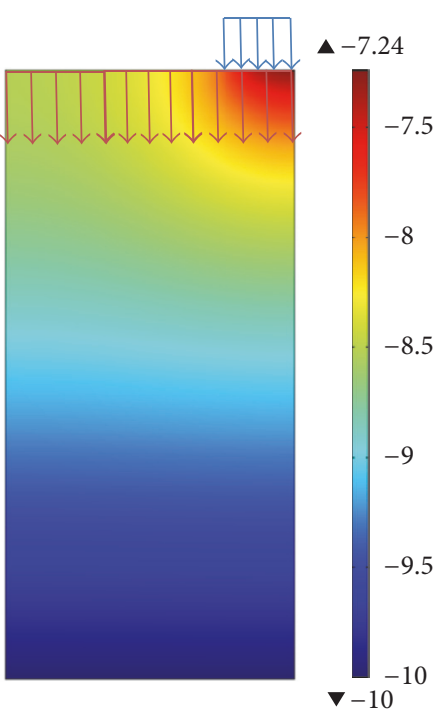

(d)

FIGURE 4: The pressure head profile variation with infiltration time: (a) $t=5 \mathrm{~h}$; (b) $t=10 \mathrm{~h}$; (c) $t=30 \mathrm{~h}$; (d) $t=50 \mathrm{~h}$. 


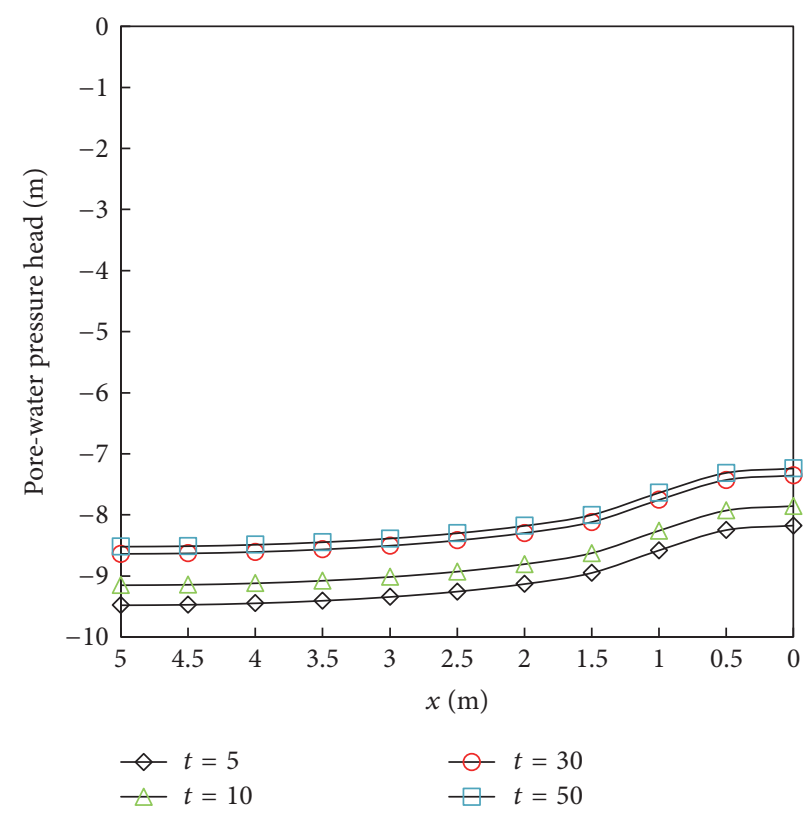

FIGURE 5: The pore-water pressure head at the boundary $0 \mathrm{~B}$ with infiltration time.

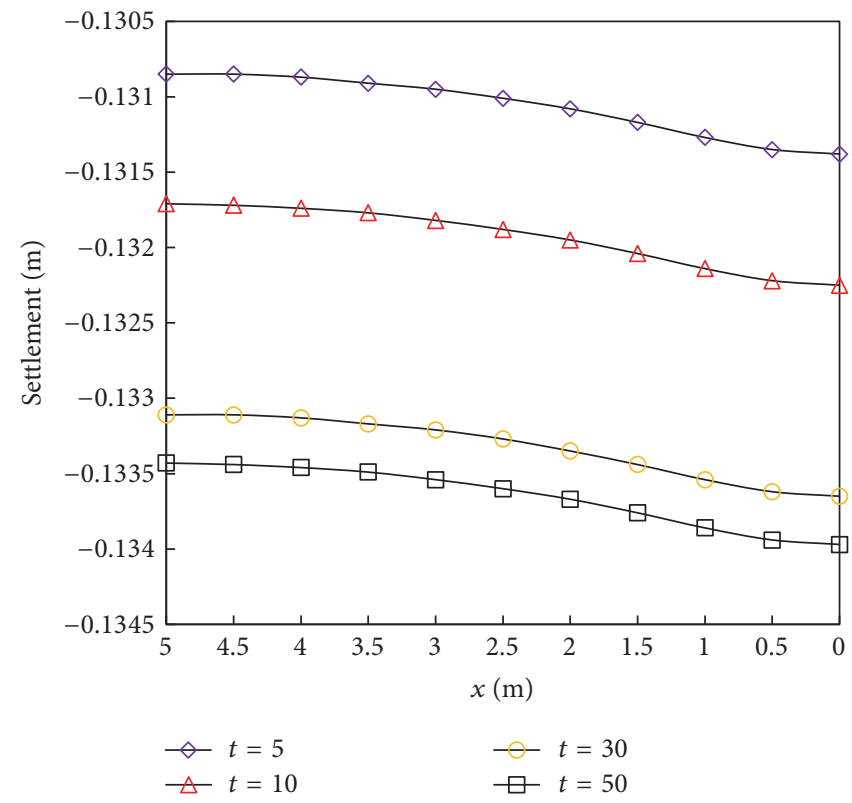

FIgURE 6: The surface settlement at the boundary $0 \mathrm{~B}$ with infiltration time.

remain unchanged. The boundary effect caused by different infiltration distances varies with the infiltration time. As shown in Figure 7, compared with $w=30 \mathrm{~m}$, the difference in the pressure head is $13 \%$ for an infiltration width of $10 \mathrm{~m}$ and $20 \%$ for a $5 \mathrm{~m}$ width at $t=10 \mathrm{~h}$. However, the difference in the pressure head is only $2 \%$ when the width of the infiltration area is $20 \mathrm{~m}$. In comparison with the $30 \mathrm{~m}$ width, the difference in the pressure head at $t=40 \mathrm{~h}$ is $17 \%$ for an infiltration width of $10 \mathrm{~m}$ and $25 \%$ for a $5 \mathrm{~m}$ width. At $t=200 \mathrm{~h}$ the difference in the pore-water pressure head for a $5 \mathrm{~m}$ width is $13 \%$ and $25 \%$ for an infiltration width of $5 \mathrm{~m}$. However, the difference in the pressure head is only $3 \%$ when the width of the infiltration area is $20 \mathrm{~m}$ at $t=50,200 \mathrm{~h}$. Figure 7 indicates that the boundary dimensions in the model play a significant role in the pore-water pressure head movement in the $2 \mathrm{D}$ semi-finite domain. It is recommended that in the computational model a width of $20 \mathrm{~m}$ (dimensionless length of 20) be used to analyze the $2 \mathrm{D}$ semi-infinite coupling problem.

Figure 8 shows the settlement at point 0 on the ground surface with the same rainfall duration. There is obvious settlement at the early stage of rainfall infiltration and the settlement rate gradually slows over time. When $k_{s}=1 \times$ $10^{-4} \mathrm{~m} / \mathrm{s}$ representing for a typical sandy soil, after $t=9 \mathrm{~h}$, the settlement of the ground surface appears to have stabilized. 


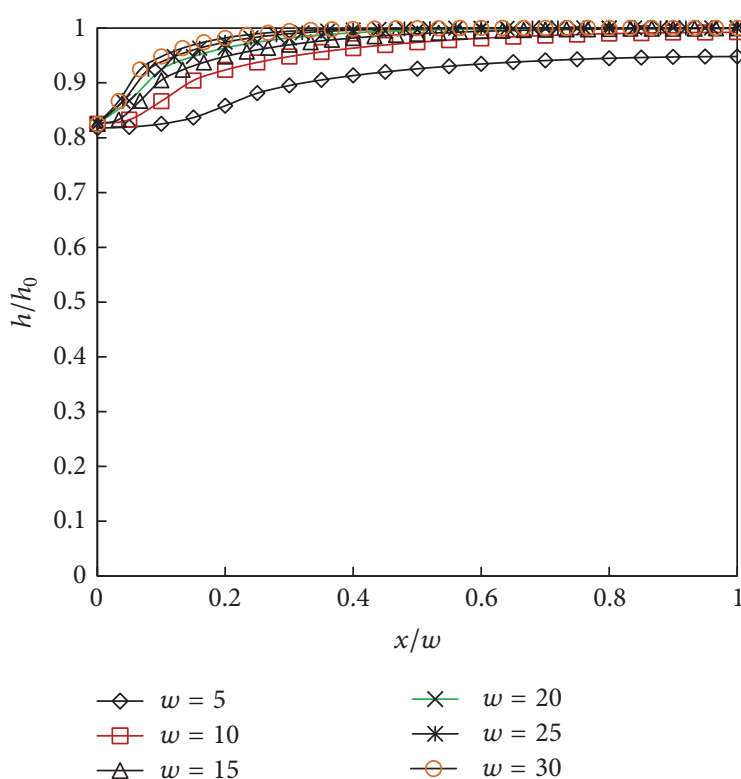

(a)

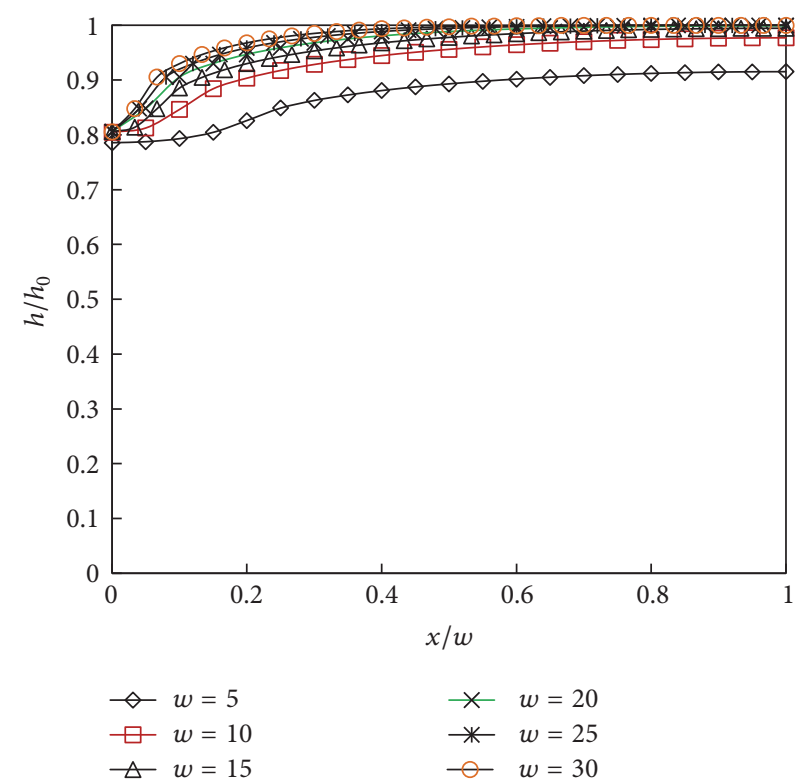

(b)

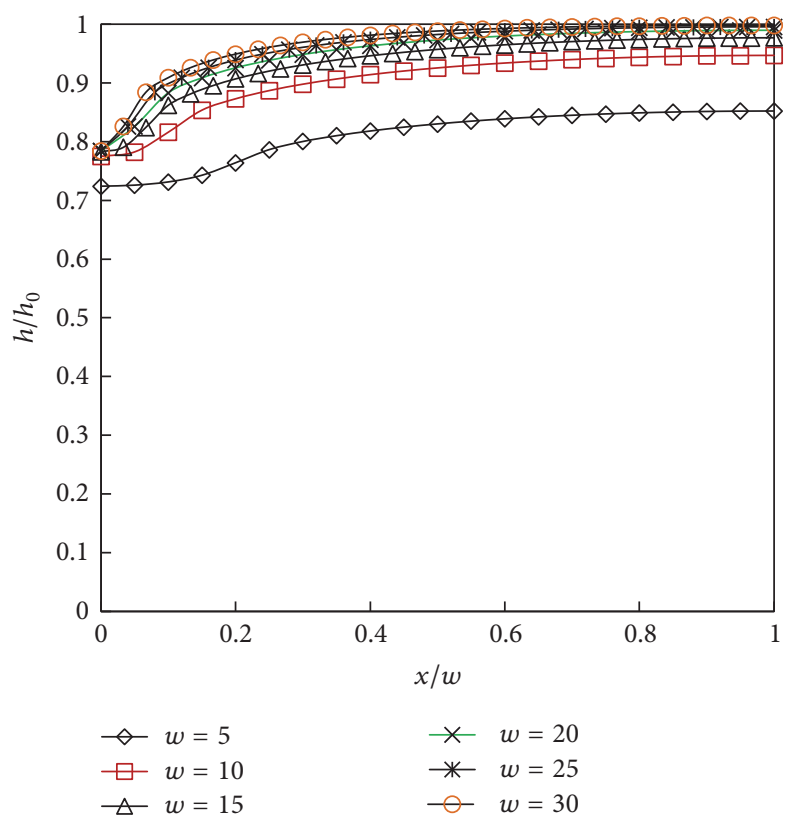

(c)

FIgURE 7: The effect of infiltration width on the pore-water pressure head at the boundary 0B: (a) $t=5 \mathrm{~h}$; (b) $t=10 \mathrm{~h}$; (c) $t=50 \mathrm{~h}$.

When $k_{s}=1 \times 10^{-5} \mathrm{~m} / \mathrm{s}$ for a typical silty soil, after $t=80 \mathrm{~h}$, the settlement of the ground surface appears to have stabilized. When $k_{s}=1 \times 10^{-6} \mathrm{~m} / \mathrm{s}$ (clay), after $t=550 \mathrm{~h}$, the settlement of the ground surface appears to have stabilized. With a decrease of $k_{s}$, the time required for stabilization becomes larger. When $k_{s} \geq 1 \times 10^{-3} \mathrm{~m} / \mathrm{s}$, the settlement at point 0 follows the same trend as $k_{s}=1 \times 10^{-4} \mathrm{~m} / \mathrm{s}$. However, the settlement for $k_{\mathrm{s}} \geq 1 \times 10^{-3} \mathrm{~m} / \mathrm{s}$ is much less that for $k_{\mathrm{s}}<1$ $\times 10^{-3} \mathrm{~m} / \mathrm{s}$.

\section{Conclusions}

A numerical method is employed to examine coupled infiltration and deformation in a semi-infinite unsaturated porous medium. The hydromechanical coupling in the semi-infinite unsaturated porous medium region could be transferred to a symmetric finite domain problem by setting up an effective investigation area. The results indicate that the numerical solutions could effectively resolve the semi-infinite 


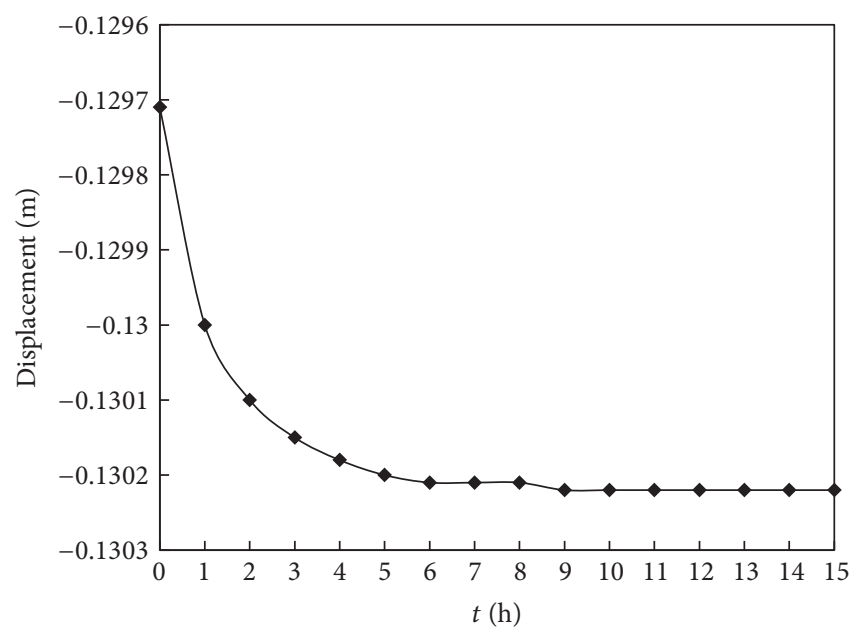

(a)

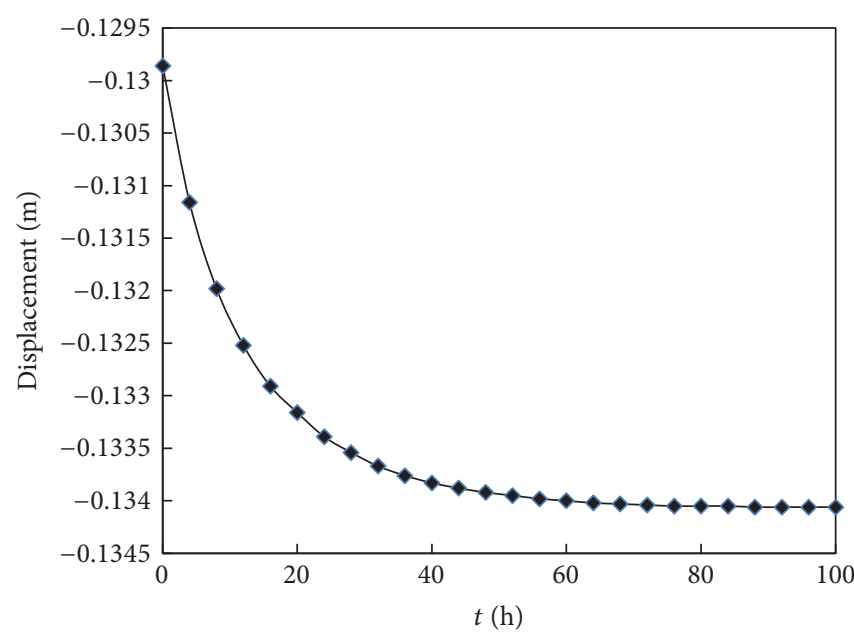

(b)

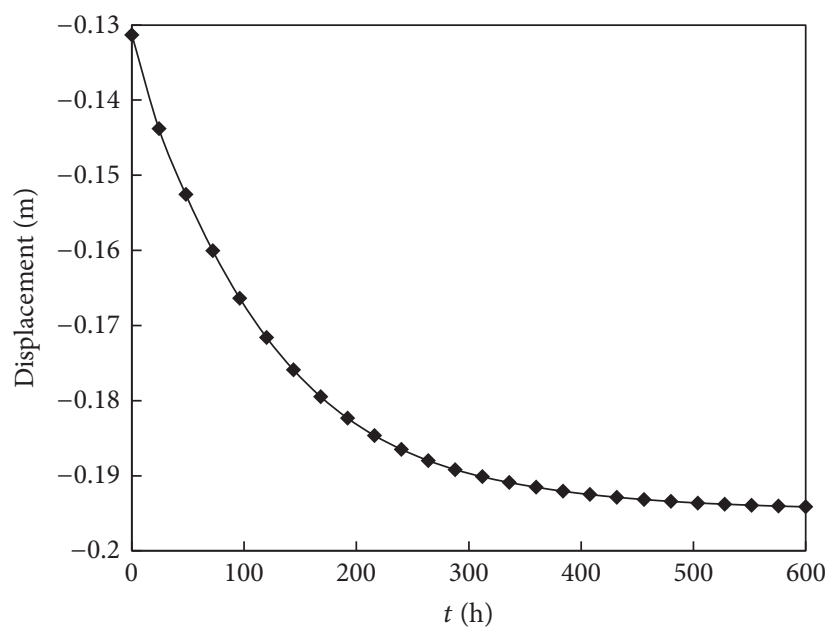

(c)

FIGURE 8: Settlement of point 0 in the model: (a) $k_{s}=1 \times 10^{-4} \mathrm{~m} / \mathrm{s}$; (b) $k_{s}=1 \times 10^{-5} \mathrm{~m} / \mathrm{s}$; (c) $k_{s}=1 \times 10^{-6} \mathrm{~m} / \mathrm{s}$.

unsaturated infiltration when the coupled problem is examined. The different widths and depths considered are of significance when examining the hydromechanical coupling during rainfall infiltration into a semi-infinite domain. The results show that the coupled effect is significantly affected by the boundary size, and the coupling is related to the duration of infiltration. The rate of change in the pore-water pressure head and settlement in an axisymmetric semi-infinite unsaturated porous medium gradually slows over the infiltration duration. This offers a first step necessary to develop more complex models describing irreversible processes of unsaturated porous media.

\section{Conflicts of Interest}

The authors declare that there are no conflicts of interest regarding the publication of this paper.

\section{Acknowledgments}

The authors acknowledge the Creative Research Groups of China (no. 41521002), the National Natural Science Foundation of China (no. 41672282), and the State Key Laboratory of Geohazard Prevention and Geoenvironment Protection (no. SKLGP2016Z017). The first author would also like to acknowledge the Environmental Geomechanics Research Group of the Department of Civil Engineering and Applied Mechanics, McGill University, for the hospitality during a research visit.

\section{References}

[1] L. Thorel, V. Ferber, B. Caicedo, and I. M. Khokhar, "Physical modelling of wetting-induced collapse in embankment base," Géotechnique, vol. 61, no. 5, pp. 409-420, 2011. 
[2] G. M. Rotisciani, G. Sciarra, F. Casini, and A. Desideri, "Hydromechanical response of collapsible soils under different infiltration events," International Journal for Numerical and Analytical Methods in Geomechanics, vol. 39, no. 11, pp. 1212-1234, 2015.

[3] J. M. Castelblanco, P. Delage, J. Pereira, and Y. Cui, "Some aspects of the compression and collapse behaviour of an unsaturated natural loess," Géotechnique Letters, vol. 1, no. 2, pp. 1722, 2011.

[4] F. Casini, "Deformation induced by wetting: a simple model," Canadian Geotechnical Journal, vol. 49, no. 8, pp. 954-960, 2012.

[5] R. Srivastava and T. C. J. Yeh, "Analytical solutions for one-dimensional, transient infiltration toward the water table in homogeneous and layered soils," Water Resources Research, vol. 27, no. 5, pp. 753-762, 1991.

[6] H. A. Basha and A. P. S. Selvadurai, "Heat-induced moisture transport in the vicinity of a spherical heat source," International Journal for Numerical and Analytical Methods in Geomechanics, vol. 22, no. 12, pp. 969-981, 1998.

[7] H. A. Basha, "Multidimensional linearized nonsteady infiltration with prescribed boundary conditions at the soil surface," Water Resources Research, vol. 35, no. 1, pp. 75-83, 1999.

[8] H. A. Basha, "Multidimensional linearized nonsteady infiltration toward a shallow water table," Water Resources Research, vol. 36, no. 9, pp. 2567-2573, 2000.

[9] J.-M. Chen, Y.-C. Tan, C.-H. Chen, and J.-Y. Parlange, "Analytical solutions for linearized Richards equation with arbitrary time-dependent surface fluxes," Water Resources Research, vol. 37, no. 4, pp. 1091-1093, 2001.

[10] R. Q. Huang and L. Z. Wu, "Analytical solutions to 1-D horizontal and vertical water infiltration in saturated/unsaturated soils considering time-varying rainfall," Computers \& Geosciences, vol. 39, pp. 66-72, 2012.

[11] L. Z. Wu, L. M. Zhang, and R. Q. Huang, "Analytical solution to $1 \mathrm{D}$ coupled water infiltration and deformation in two-layer unsaturated soils," International Journal for Numerical and Analytical Methods in Geomechanics, vol. 36, no. 6, pp. 798-816, 2012.

[12] T. L. T. Zhan, G. W. Jia, Y.-M. Chen, D. G. Fredlund, and H. $\mathrm{Li}$, "An analytical solution for rainfall infiltration into an unsaturated infinite slope and its application to slope stability analysis," International Journal for Numerical and Analytical Methods in Geomechanics, vol. 37, no. 12, pp. 1737-1760, 2013.

[13] L. Z. Wu, A. P. S. Selvadurai, L. M. Zhang, R. Q. Huang, and J. Huang, "Poro-mechanical coupling influences on potential for rainfall-induced shallow landslides in unsaturated soils," Advances in Water Resources, vol. 98, pp. 114-121, 2016.

[14] L. Z. Wu and L. M. Zhang, "Analytical solution to 1D coupled water infiltration and deformation in unsaturated soils," International Journal for Numerical and Analytical Methods in Geomechanics, vol. 33, no. 6, pp. 773-790, 2009.

[15] L. Z. Wu, P. Sun, and R. Q. Huang, "Analytical Analysis of Partially Saturated Infiltration Coupled with Deformation in a Semi-Infinite Region," International Journal of Geomechanics, vol. 17, no. 6, p. 06016040, 2017.

[16] L. Z. Wu, L. M. Zhang, Y. Zhou, and B. E. Li, "Analysis of multiphase coupled seepage and stability in anisotropic slopes under rainfall condition," Environmental Earth Sciences, vol. 76, no. 14, article 469, 2017.

[17] E. Garcia, F. Oka, and S. Kimoto, "Numerical analysis of a onedimensional infiltration problem in unsaturated soil by a seepage-deformation coupled method," International Journal for
Numerical and Analytical Methods in Geomechanics, vol. 35, no. 5, pp. 544-568, 2011.

[18] N. Khalili and S. Zargarbashi, "Influence of hydraulic hysteresis on effective stress in unsaturated soils," Géotechnique, vol. 60, no. 9, pp. 729-734, 2010.

[19] E. E. Alonso, A. Gens, and A. Josa, "A constitutive model for partially saturated soils," Géotechnique, vol. 40, no. 3, pp. 405430, 1990.

[20] W. Ehlers, T. Graf, and M. Ammann, "Deformation and localization analysis of partially saturated soil," Computer Methods Applied Mechanics and Engineering, vol. 193, no. 27-29, pp. 28852910, 2004.

[21] G. M. Rotisciani, F. Casini, A. Desideri, and G. Sciarra, "Hydromechanical behavior of an embankment during inundation," Canadian Geotechnical Journal, vol. 54, no. 3, pp. 348-358, 2017.

[22] S. E. Cho and S. R. Lee, "Instability of unsaturated soil slopes due to infiltration," Computers \& Geotechnics, vol. 28, pp. 185208, 2001.

[23] E. E. Alonso, A. Gens, and C. H. Delahaye, "Influence of rainfall on the deformation and stability of a slope in overconsolidated clays: a case study," Hydrogeology Journal, vol. 11, no. 1, pp. 174192, 2003.

[24] D. G. Fredlund and H. Rahardjo, Soil Mechanics for Unsaturated Soils, John Wiley and Sons, New York, NY, USA, 1993.

[25] N. Khalili, M. A. Habte, and S. Zargarbashi, "A fully coupled flow deformation model for cyclic analysis of unsaturated soils including hydraulic and mechanical hystereses," Computers \& Geosciences, vol. 35, no. 6, pp. 872-889, 2008.

[26] S. J. Wheeler, R. S. Sharma, and M. S. R. Buisson, "Coupling of hydraulic hysteresis and stress-strain behaviour in unsaturated soils," Géotechnique, vol. 53, no. 1, pp. 41-54, 2003.

[27] R. O. Davis and A. P. S. Selvadurai, Elasticity and Geomechanics, Cambridge University Press, Cambridge, UK, 1996.

[28] M. T. van Genuchten, "A closed-form equation for predicting the hydraulic conductivity of unsaturated soils," Soil Science Society of America Journal, vol. 44, no. 5, pp. 892-898, 1980.

[29] V. Dakshanamurthy, D. G. Fredlund, and H. Rahardjo, "Coupled three-dimensional consolidation theory of unsaturated porous media," in Proceedings of the International Conference on Expansive Soils, pp. 99-103, 1984.

[30] R. O. Davis and A. P. S. Selvadurai, Plasticity and Geomechanics, Cambridge University Press, Cambridge, UK, 2004.

[31] S. Pietruszczak, Fundamentals of Plasticity in Geomechanics, CRC Press, Boca Raton, Fla, USA, 2010.

[32] A. P. Selvadurai and A. P. Suvorov, "Boundary heating of poroelastic and poro-elasto-plastic spheres," Proceedings of the Royal Society A Mathematical, Physical and Engineering Sciences, vol. 468, no. 2145, pp. 2779-2806, 2012.

[33] A. P. S. Selvadurai and A. P. Suvorov, "Thermo-poromechanics of a fluid-filled cavity in a fluid-saturated geomaterial," Proceedings of the Royal Society A Mathematical, Physical and Engineering Sciences, vol. 470, no. 2163, 2014.

[34] W. R. Gardner, "Some steady-state solutions of the unsaturated moisture flow equation with application to evaporation from a water table," Soil Science, vol. 85, no. 4, pp. 228-232, 1958.

[35] M. Ozisik, Boundary Value Problems of Heat Conduction, Dover, New York, NY, USA, 1989.

[36] L. Z. Wu and A. P. S. Selvadurai, "Rainfall infiltration-induced groundwater table rise in an unsaturated porous medium," Environmental Earth Sciences, vol. 75, no. 2, pp. 1-11, 2016. 

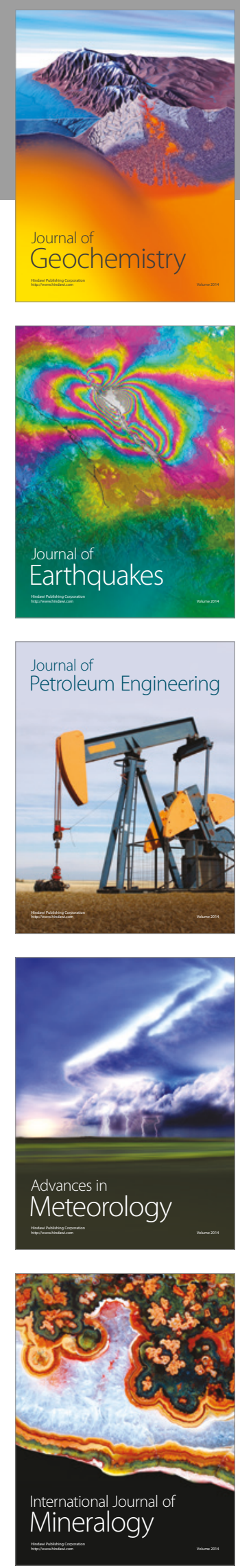
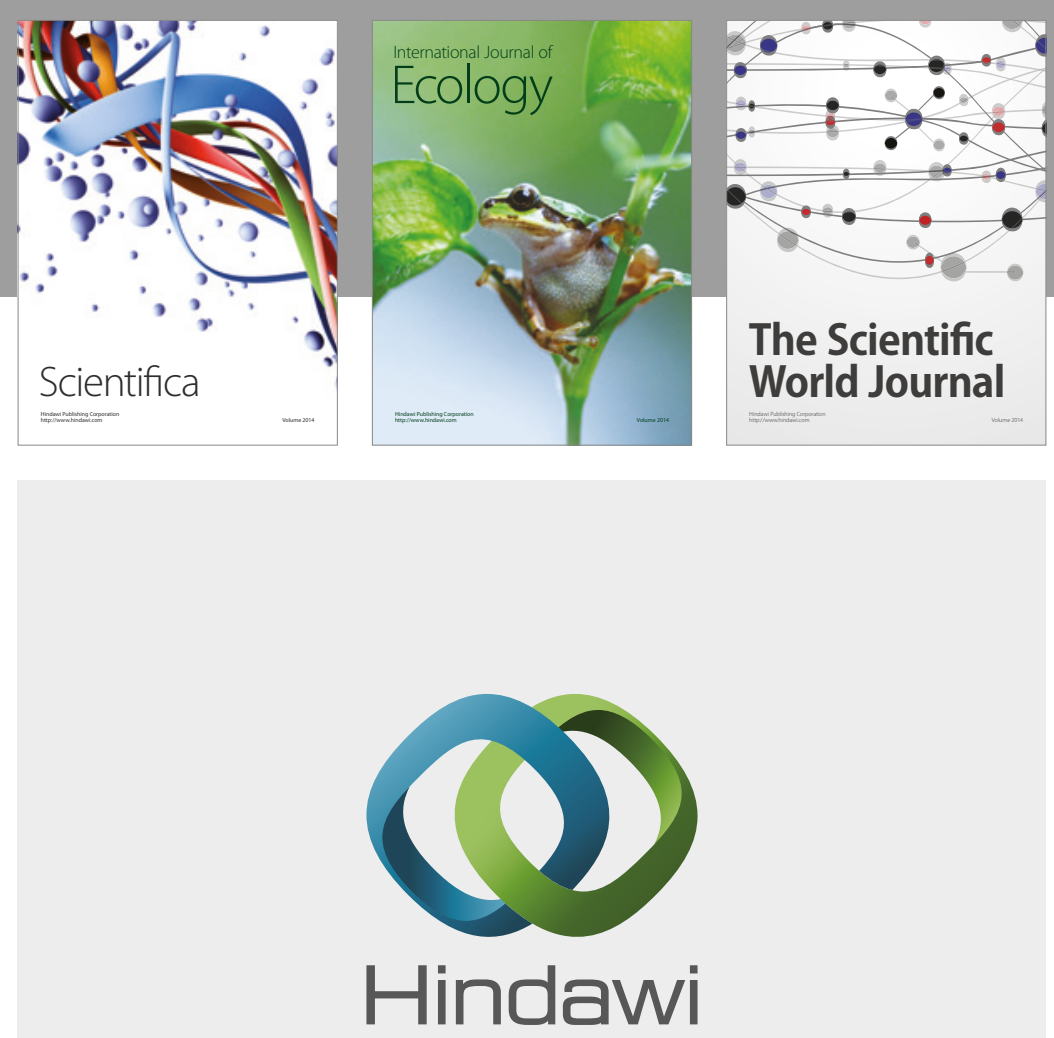

Submit your manuscripts at

https://www.hindawi.com
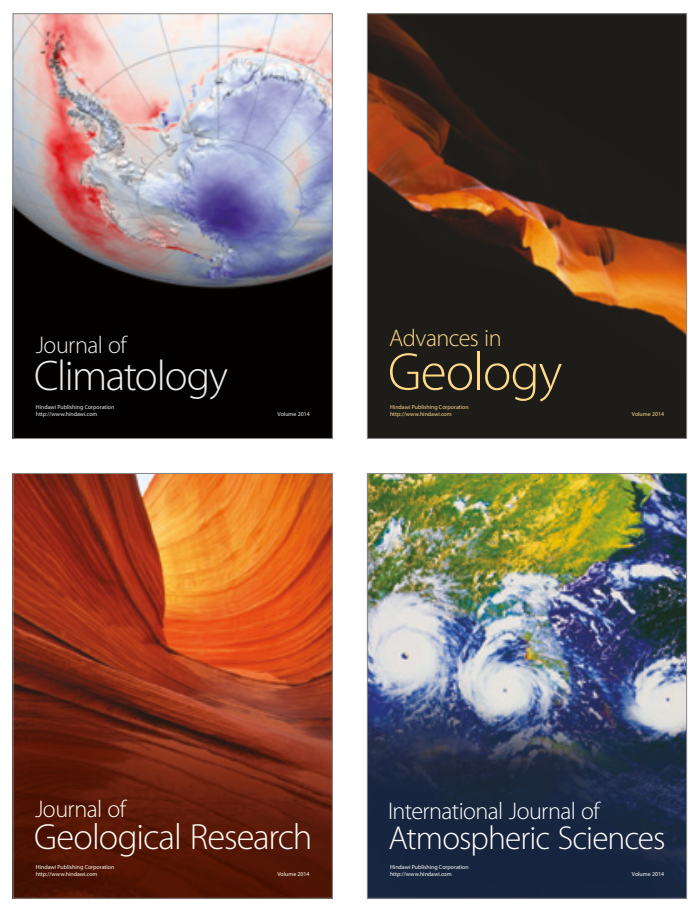

The Scientific

World Journal
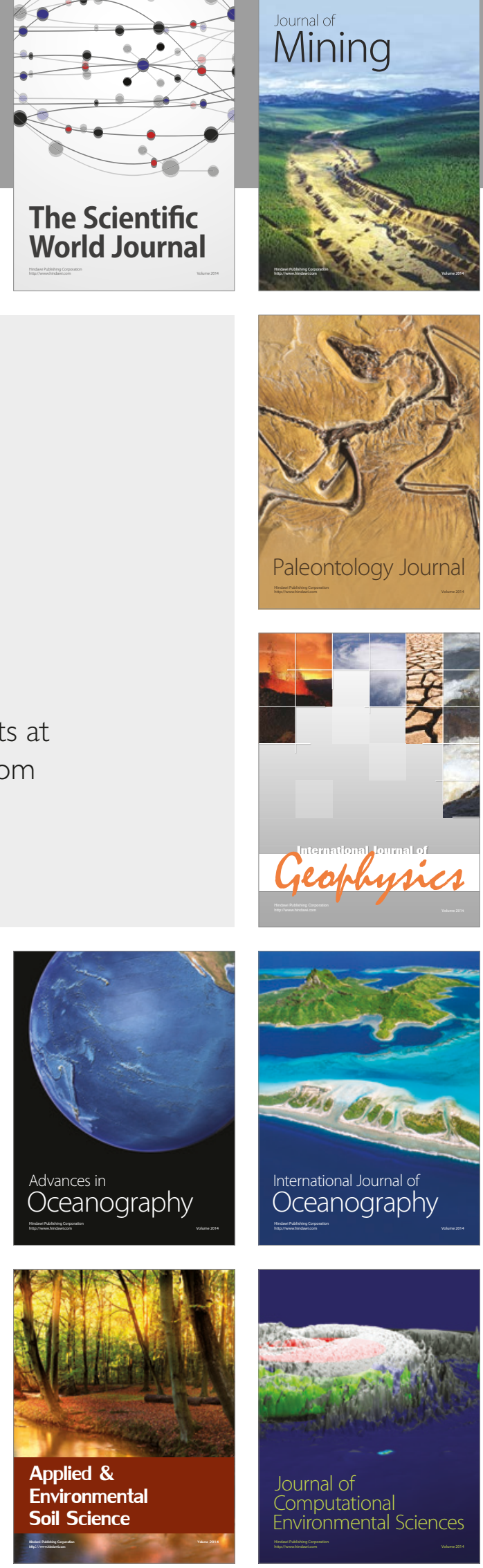